\title{
The impact of intratumoral metabolic heterogeneity on postoperative recurrence and survival in resectable esophageal squamous cell carcinoma
}

\author{
Xinzhe Dong ${ }^{1,2}$, Xiaorong Sun ${ }^{3}$, Xianguang Zhao ${ }^{1}$, Wanqi Zhu ${ }^{1}$, Lu Sun ${ }^{4}$, Yong Huang ${ }^{3}$, \\ Wenwu Li ${ }^{3}$, Honglin Wan ${ }^{5}$, Ligang Xing ${ }^{1,2}$, Jinming $\mathrm{Yu}^{1,2}$ \\ ${ }^{1}$ Department of Radiation Oncology, Shandong Cancer Hospital, Shandong University, Jinan, Shandong, China \\ ${ }^{2}$ Key Laboratory of Radiation Oncology of Shandong Province, Shandong Academy of Medical Sciences, Jinan, Shandong, China \\ ${ }^{3}$ Department of Radiology, Shandong Cancer Hospital and Institute, Jinan, Shandong, China \\ ${ }^{4}$ Jinan University, Jinan, Shandong, China \\ ${ }^{5}$ College of Physics and Electronic Science, Shandong Normal University, Jinan, Shandong, China
}

Correspondence to: Ligang Xing, email: sdcancerhospital@163.com

Keywords: positron emission tomography, FDG, esophageal carcinoma, intratumoral heterogeneity, prognosis

Received: July 18, 2016 Accepted: January 10, $2017 \quad$ Published: January 19, 2017

\section{ABSTRACT}

Objective: To evaluate the impact of intratumoral metabolic heterogeneity measured by 18F-FDG PET imaging on postoperative recurrence and survival for patients with esophageal squamous cell carcinoma (ESCC).

Results: AUC-CSH, metabolic tumor volume and $\mathrm{pN}$-stage were significant prognostic factors for RFS. Additionally, tumor recurrence of the low AUC-CSH group ( $\leq 0.478)$ was 3 times higher than high group $(P=0.015)$. The median OS of patients with advanced AJCC stage or low AUC-CSH was also significantly shorter than that of patients with stage I \& II or high AUC-CSH $(P=0.021,0.009)$. Multivariate analysis identified the AUC-CSH to be the only significant risk factor for postoperative recurrence and overall survival in whole-group and stage III patients.

Materials and Methods: 116 ESCC patients who underwent staging 18F-FDG PET-CT scan and surgical resection were reviewed. The metabolic parameters were assessed as follows: maximum standardized uptake value $\left(\right.$ SUV $\left._{\max }\right)$, metabolic tumor volume, and the area under the curve of the cumulative SUV-volume histogram (AUC$\mathrm{CSH}$ ), which is known to reflect the intratumoral metabolic heterogeneity. Regression analyses were used to identify clinicopathological and imaging variables associated with relapse-free survival (RFS) and overall survival (OS).

Conclusions: Intratumoral metabolic heterogeneity characterized by AUC-CSH can predict postoperative recurrence and survival in patients with resectable ESCC.

\section{INTRODUCTION}

Apart from the high incidence rate, esophageal carcinoma is one of the sixth leading causes of cancerrelated deaths in the world [1]. It is known that Asian countries have the highest rates of esophageal cancer [2]. Compared with other types of esophageal carcinoma (mainly adenocarcinoma), esophageal squamous cell carcinoma (ESCC) has a poorer long-term survival. Esophagectomy has historically been considered the mainstay of treatment but has disappointing long-term outcomes [3].

Intratumoral heterogeneity is a well-recognized feature of malignancy tumor. A measurement that can quantify intratumoral heterogeneity may potentially predict outcome of ESCC . Positron emission tomography (PET) with 18F-fluorodeoxy glucose (18F-FDG) reflects important functional information on tumor cells. Before morphological changes occur, PET image shows metabolic abnormalities [4]. Currently, there is an increasing interest in using $18 \mathrm{~F}-\mathrm{FDG}$ PET images to quantify tumor tracer uptake heterogeneity and predicting treatment outcome non-invasively [5].

Recently, a innovative way to quantify intratumoral tracer uptake heterogeneity has been proposed by El Naqa et al. Cumulative SUV volume histogram (CSH), describes $\%$ of total tumor volume above $\%$ threshold of maximum 
SUV [6]. The area under the CSH (AUC-CSH) may be a quantitative parameter of metabolic heterogeneity. Our previous studies $[7,8]$ also showed that tumor FDG uptake heterogeneity accessed by PET image feature has the potential to detect advanced stage tumors in ESCC.

Kang. et al used AUC-CSH to define intratumoral metabolic heterogeneity of inoperable advanced nonsmall cell lung cancer (NSCLC), they found that AUC$\mathrm{CSH}$ could predict disease progression after concurrent chemoradiotherapy (CCRT) [9]. Our previous study also found that the NSCLC patients with greater intra-treatment AUCH-CSH change had significantly longer survival time [10]. However, none of these studies have attempted to quantify $18 \mathrm{~F}-\mathrm{FDG}$ uptake heterogeneity using the AUC$\mathrm{CSH}$ in patients with radical ESCC resection. Besides, the sample sizes of most similar studies were small, and the results of the prediction value of heterogeneity parameter remained undetermined.

The object of this research was to assess the possible usefulness of the intratumoral 18F-FDG uptake heterogeneity measured by PET imaging in prediction of postoperative recurrence and survival for patients with resectable ESCC.

\section{RESULTS}

\section{Clinical characteristics}

Table 1 shows patient demographics and Clinical Characteristics. The median age was 62.8 years (range 36.2-75.4), and they were followed for mean 39.6 months (range 4.6-68.0 months). Preoperative $\mathrm{SUV}_{\text {max }}$ ranged between 3.0 and 24.3, with median of 12.5. The median of MTV was $13.6 \mathrm{~cm}^{3}$ (range $3.2 \mathrm{~cm}^{3}-46.9 \mathrm{~cm}^{3}$ ). AUC-CSH ranged between 0.276 and 0.718 , with median of 0.487 . 27 patients received right-sided thoracotomy and upper midline laparotomy, other patients received left-sided thoracotomy for esophagectomy. All patients underwent $\mathrm{R} 0$ resection got a pathological stage.

The relationship between intratumoral heterogeneity status and clinicopathological characteristics is also summarized in Table 1. Lower AUC-CSH was significantly correlated with larger baseline MTV $(P=0.029)$, advanced postoperative AJCC stage $(P=0.018)$ and $\mathrm{N}$ stage $(P=0.036)$. On the other hand, there were no significant relationships between AUC$\mathrm{CSH}$ value and age, gender, treatment modality, tumor differentiation, ECOG performance status, preoperative comorbidity, location, $\mathrm{T}$ stage or $\mathrm{SUV}_{\text {max }}$.

\section{Relapse-free survival and recurrence pattern}

With a 3-year RFS of $33.6 \%$, the median RFS was $21.8 \pm 14.1$ months. Univariate survival analyses of RFS were shown in Table 2. Patients with lower value of AUC-CSH $(\leq 0.487)$ relapsed quickly than higher
AUC-CSH ( $>0.487$ ) (Median RFS: 13.4 months vs. 30.6 months, $P=0.002$, Figure 1A). Larger MTV ( $>13.6)$ was also associated with shorter relapse time $(P=0.033$; Median RFS: 22.6 vs. 15.7 months). Besides, RFS was shorter in N2 and N3 patients $(P=0.027$; Median RFS 18.8 vs. 25.6 months). As shown in multivariate COX regression analyses (Table 3), AUC-CSH was the only independent prognostic factors of RFS $(P=0.008$; HR: 3.153 95\% CI: 2.873-4.821).

Recurrence was reported in $46(39.7 \%)$ patients. On evaluating the recurrence pattern, we found 20 cases at locoregional level (12 relapse in situ, 5 mediastinal lymph nodes, and 3 abdominal lymph nodes), 17 distant cases, and 9 cases of mixed recurrences. Besides, tumor recurrence was observed in 35 (59.3\%) of the low AUC$\mathrm{CSH}$ group $(\leq 0.478)$, which was 3 times higher than high AUC-CSH group $(P=0.015)$; In particular, locoregional recurrence showed a significantly higher rate in the low AUC-CSH group $(P=0.001)$. The frequencies of distant metastasis in the low AUC-CSH group were more than twice higher than in the high AUC-CSH group (Table 4).

\section{Overall survival analysis}

In this study, 35.3 percent of patients were alive three years later, the median OS was $27.7 \pm 20.2$ months. Univariate analyses revealed that preoperative clinical features and treatment modality were not risk factors of OS (Table 2). On the other hand, lower AUC-CSH $(\leq 0.487)$ was a significantly predictor of shorter OS (median OS: 18.4 vs. 37.2 months $P=0.004$, Figure 1B). The overall survival of advanced AJCC stage (III-IV) patients was also shorter than stage I\&II (median OS: 25.6 vs. 34.9 months $P=0.027)$. Besides, $\mathrm{pT}$ stage, $\mathrm{pN}$ stage and histologic grade were also not significant predictors of OS (Table 2). Median OS of patients with lower MTV $(\leq 13.6)$ was not significantly longer than high MTV ( $>13.6)$ (28.1 vs. 29.3 months $P=0.083$ ).

Each of clinical and PET features entered multivariate analysis separately, for high degree of collinearity with each other. At multivariate Cox analysis for OS outcome (Table 3), it was found that the only independent predictive factor associated with decreased overall survival was low AUC-CSH $(P=0.016$; $H R=3.062,95 \%$ CI: 2.277-4.879).

In the subgroup analysis of pathologic stage III patients, the median of AUC-CSH is 0.521. Compared with high AUC-CSH, patients with lower AUC-CSH value progressed quickly and had a significantly shorter survival time $(P=0.023$ and $P=0.012$, Figure 1C, 1D). After multivariable analysis, only AUC-CSH retained its significant predictive value for RPS $(P=0.032, H R=2.586$, 95\% CI: $1.952-4.273)$ and $\mathrm{OS}(P=0.026 ; H R=3.215,95 \%$ CI: 2.526-5.628). High pN-stage, MTV or AJCC stage (III, III $_{\mathrm{b}}$ vs. III $)(P=0.079, P=0.143$ and $P=0.239)$ were not independent prognostic factors for shorter RFS or poor OS. 
Table 1: Patient demographics and clinical characteristics of 116 ESCC

\begin{tabular}{|c|c|c|c|}
\hline Characteristics & No. of patients $(\%)$ & AUC-CSH (mean \pm SD) & $P$ value \\
\hline Age & & & 0.289 \\
\hline $\operatorname{High}(>62.8)$ & $52(44.8 \%)$ & $0.503 \pm 0.108$ & \\
\hline Low $(\leq 62.8)$ & $64(55.2 \%)$ & $0.472 \pm 0.085$ & \\
\hline Sex & & & 0.894 \\
\hline Male & $96(82.7 \%)$ & $0.467 \pm 0.087$ & \\
\hline Female & $20(17.3 \%)$ & $0.585 \pm 0.076$ & \\
\hline Tumor location & & & 0.524 \\
\hline Upper & $16(13.8 \%)$ & $0.461 \pm 0.082$ & \\
\hline Middle & $66(56.9 \%)$ & $0.591 \pm 0.075$ & \\
\hline Lower & $34(29.3 \%)$ & $0.474 \pm 0.093$ & \\
\hline ECOG performance status & & & 0.068 \\
\hline 0 & $13(11.2 \%)$ & $0.431 \pm 0.062$ & \\
\hline 1 & $99(85.3 \%)$ & $0.495 \pm 0.099$ & \\
\hline 2 & $4(3.5 \%)$ & $0.471 \pm 0.096$ & \\
\hline Preoperative comorbidity & & & 0.195 \\
\hline No/minor comorbidity & $104(89.7 \%)$ & $0.483 \pm 0.098$ & \\
\hline Major comorbidity & $12(10.3 \%)$ & $0.521 \pm 0.074$ & \\
\hline SUVmax & & & 0.068 \\
\hline $\operatorname{High}(>12.5)$ & $57(49.1 \%)$ & $0.431 \pm 0.060$ & \\
\hline Low $(\leq 12.5)$ & $59(50.8 \%)$ & $0.521 \pm 0.062$ & \\
\hline MTV & & & 0.029 \\
\hline $\operatorname{High}\left(>13.6 \mathrm{~cm}^{3}\right)$ & $53(45.7 \%)$ & $0.407 \pm 0.061$ & \\
\hline Low $\left(\leq 13.6 \mathrm{~cm}^{3}\right)$ & $63(54.3 \%)$ & $0.593 \pm 0.066$ & \\
\hline Treatment modality & & & 0.129 \\
\hline Surgery alone & $9(7.7 \%)$ & $0.331 \pm 0.042$ & \\
\hline Surgery + adjuvant RT & $38(32.8 \%)$ & $0.527 \pm 0.124$ & \\
\hline Surgery + adjuvant CT & $14(12.1 \%)$ & $0.532 \pm 0.032$ & \\
\hline Surgery + adjuvant RT $+\mathrm{CT}$ & $55(47.4 \%)$ & $0.463 \pm 0.142$ & \\
\hline pT stage & & & 0.079 \\
\hline $\mathrm{T} 1$ & $22(14.3 \%)$ & $0.521 \pm 0.096$ & \\
\hline $\mathrm{T} 2$ & $24(20.2 \%)$ & $0.522 \pm 0.077$ & \\
\hline $\mathrm{T} 3$ & $39(43.3 \%)$ & $0.458 \pm 0.106$ & \\
\hline $\mathrm{T} 4$ & $31(22.2 \%)$ & $0.493 \pm 0.090$ & \\
\hline $\mathrm{pN}$ stage & & & 0.018 \\
\hline No & $39(33.6 \%)$ & $0.505 \pm 0.082$ & \\
\hline N1 & $29(25.0 \%)$ & $0.495 \pm 0.114$ & \\
\hline N2 & $31(26.7 \%)$ & $0.385 \pm 0.096$ & \\
\hline N3 & $17(14.7 \%)$ & $0.369 \pm 0.065$ & \\
\hline pM stage & & & 0.092 \\
\hline M0 & $110(94.8 \%)$ & $0.490 \pm 0.097$ & \\
\hline M1 & $6(5.2 \%)$ & $0.422 \pm 0.080$ & \\
\hline pG stage & & & 0.052 \\
\hline G1 & $24(20.7 \%)$ & $0.522 \pm 0.077$ & \\
\hline $\mathrm{G} 2$ & $62(53.4 \%)$ & $0.482 \pm 0.091$ & \\
\hline G3 & $30(25.9 \%)$ & $0.465 \pm 0.104$ & \\
\hline pAJCC stage & & & 0.036 \\
\hline I & $20(17.2 \%)$ & $0.538 \pm 0.084$ & \\
\hline II & $22(19.1 \%)$ & $0.515 \pm 0.094$ & \\
\hline III & $68(58.6 \%)$ & $0.469 \pm 0.095$ & \\
\hline IV & $6(0.5 \%)$ & $0.382 \pm 0.080$ & \\
\hline
\end{tabular}


Table 2: Univariate survival analyses of factors associated with RFS and OS

\begin{tabular}{|c|c|c|c|c|c|c|}
\hline \multirow{2}{*}{ Variable } & \multicolumn{3}{|c|}{ Relapse-free survival } & \multicolumn{3}{|c|}{ Overall survival } \\
\hline & HR & $95 \% \mathrm{CI}$ & $P$ value & HR & $95 \% \mathrm{CI}$ & $P$ value \\
\hline Age $(\geq 62)$ & 2.987 & $0.839-8.583$ & 0.542 & 3.453 & $0.452-6.217$ & 0.361 \\
\hline Sex (male vs. female) & 1.797 & $0.238-5.380$ & 0.876 & 2.832 & $0.523-3.401$ & 0.526 \\
\hline $\begin{array}{l}\text { Histologic grade } \\
\text { (G3 vs. G2, G1) }\end{array}$ & 1.146 & $0.786-1.670$ & 0.479 & 2.042 & $0.323-5.737$ & 0.242 \\
\hline ECOG (2 vs. 1,0$)$ & 2.165 & $0.829-5.438$ & 0.326 & 1.883 & $0.783-3.264$ & 0.682 \\
\hline $\begin{array}{l}\text { Comorbidity } \\
\text { (major vs. no, minor) }\end{array}$ & 1.672 & $0.659-2.341$ & 0.563 & 1.361 & $0.406-3.726$ & 0.421 \\
\hline $\begin{array}{l}\text { Tumor location } \\
\text { (upper vs. other) }\end{array}$ & 1.575 & $0.748-3.316$ & 0.231 & 1.133 & $0.248-2.138$ & 0.163 \\
\hline SUVmax $(>12.5)$ & 3.505 & $0.233-12.761$ & 0.365 & 4.221 & $0.683-7.294$ & 0.365 \\
\hline $\operatorname{MTV}\left(>13.6 \mathrm{~cm}^{3}\right)$ & 3.197 & $1.904-5.376$ & 0.033 & 1.173 & $0.913-1.358$ & 0.083 \\
\hline $\begin{array}{l}\text { Surgery alone } \\
\text { (vs. adjuvant therapy) }\end{array}$ & 0.484 & $0.193-1.210$ & 0.121 & 1.335 & $0.561-3.178$ & 0.513 \\
\hline T4, T3 (vs. T2, T1) & 0.237 & $0.032-1.771$ & 0.161 & 2.433 & $1.003-5.899$ & 0.049 \\
\hline N3, N2 (vs. N1, N0) & 1.956 & $1.522-2.749$ & 0.027 & 2.558 & $1.415-4.624$ & 0.071 \\
\hline M1 (vs. M0) & 1.134 & $0.587-2.193$ & 0.708 & 1.567 & $0.808-2.033$ & 0.101 \\
\hline $\begin{array}{l}\text { AJCC stage III, IV } \\
\text { (vs. I, II) }\end{array}$ & 2.022 & $0.836-4.890$ & 0.098 & 2.397 & $1.177-4.879$ & 0.027 \\
\hline AUC-CSH $(\leq 0.478)$ & 3.529 & $2.118-6.892$ & 0.002 & 2.745 & $1.506-4.938$ & 0.004 \\
\hline
\end{tabular}

A

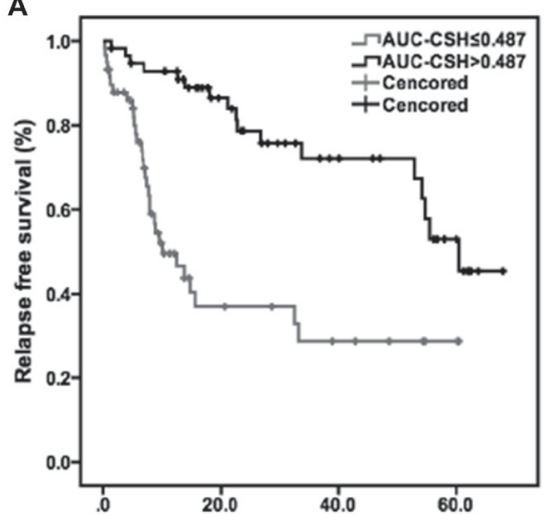

C

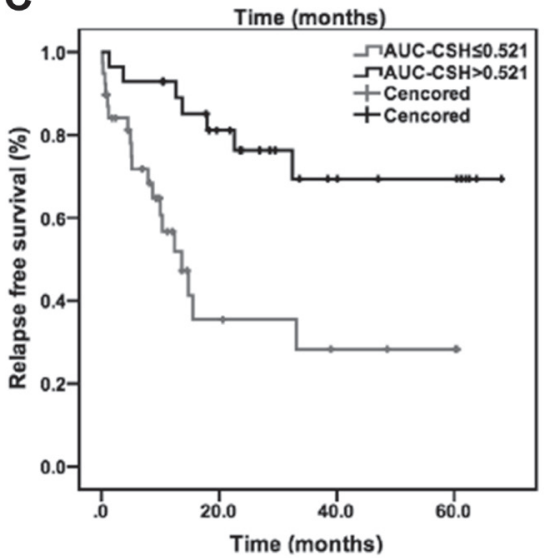

B

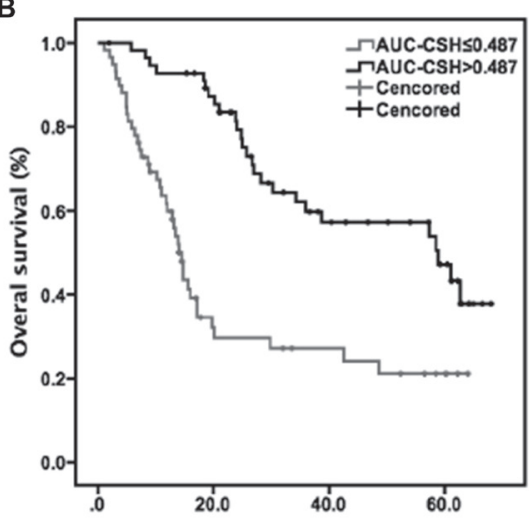

D

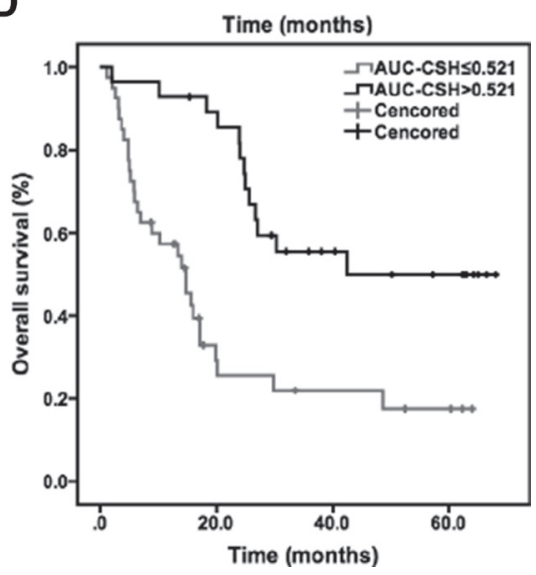

Figure 1: Kaplan-Meier plots for probability of RFS and OS. Kaplan-Meier analyses of relapse free survival and overall survival according to AUC-CSH in whole group (116 patients) and subgroup (stage III, 58 patients). 
Table 3: Multivariate survival analyses of factors associated with RFS and OS

\begin{tabular}{|c|c|c|c|c|c|c|}
\hline \multirow{2}{*}{ Variable } & \multicolumn{2}{|c|}{ Relapse-free survival } & \multirow{2}{*}{$P$ value } & \multicolumn{2}{|c|}{ Overall survival } & \multirow{2}{*}{$P$ value } \\
\hline & HR & $95 \% C I$ & & $\overline{H R}$ & $95 \% C I$ & \\
\hline $\operatorname{MTV}\left(>13.6 \mathrm{~cm}^{3}\right)$ & 1.236 & $0.719-1.852$ & 0.433 & 1.573 & $0.629-1.953$ & 0.326 \\
\hline N3, N2 (vs. N1, N0) & 1.956 & $0.532-1.169$ & 0.268 & 2.527 & $0.915-4.265$ & 0.202 \\
\hline AJCC stage III, IV (vs. I, II) & 2.193 & $0.431-4.245$ & 0.118 & 1.236 & $0.749-3.126$ & 0.241 \\
\hline AUC-CSH $(\leq 0.478)$ & 3.153 & $2.873-4.821$ & 0.008 & 3.062 & $2.277-4.879$ & 0.016 \\
\hline
\end{tabular}

Table 4: Patterns of recurrence

\begin{tabular}{lcccc}
\hline \multicolumn{1}{c}{ Recurrence pattern } & No. of Patients (\%) & High AUC-CSH (\%) & Low AUC-CSH (\%) & $\boldsymbol{P}_{\text {value }}$ \\
\hline Total recurrence & $46(39.7 \%)$ & $11(19.3 \%)$ & $35(59.3 \%)$ & 0.015 \\
Locoregional recurrence & $20(43.5 \%)$ & $5(8.8 \%)$ & $15(25.4 \%)$ & 0.001 \\
Distant metastasis & $17(36.9 \%)$ & $5(8.8 \%)$ & $12(20.3 \%)$ & 0.023 \\
Mixed recurrences & $9(19.6 \%)$ & $4(7.0 \%)$ & $5(8.5 \%)$ & 0.279 \\
\hline
\end{tabular}

\section{DISCUSSION}

Personalized medicine is a goal in modern cancer therapy that aims for optimal treatment for an individual patient that is dependent on tumor characteristics in that individual [11]. Despite medical advances in recent decades for the treatment of esophageal carcinoma, long term survival rates are still low [12]. It is therefore important to identify better prognostic factors. This study showed that intratumoral metabolic heterogeneity assessed by AUC-CSH is a strong independent prognostic feature in patients with ESCC resection.

Our study analyzed clinical and pathologic features of 116 resectable ESCC, the prognostic value of AUC$\mathrm{CSH}$ was confirmed. A lower baseline AUC-CSH value (higher metabolic heterogeneity status) was correlated with poor outcome. Besides that, in the subgroup (III stage) analysis, the AUC-CSH was also an accurate predictor of RFS or OS. As it is found that most esophagus carcinoma is not discovered until the advanced stages, the observation is more valuable. In addition to pathological AJCC stage, AUC-CSH might be a noninvasive quantitative biomarker to stratify patients with a worse outcome before treatment is initiated.

According to results of our previous [10] and current studies, AUC-CSH was related to AJCC stage. Moreover, multifactor analysis revealed that AUC-CSH was an independent prognostic factor of survival. This observation may have a reasonable explanation that reflects the inherent biologic characteristics of the tumors [13]. The patients with higher heterogeneous tumors fare more poorly than those with homogeneous tumors, and accompany with higher lymph node metastasis rate.

This result is not unexpected because intratumor heterogeneity may foster tumor adaptation and therapeutic failure through Darwinian selection [14]. Aggressive tumors grow faster and involve more intratumoral hypoxia [15], which leads to FDG accumulate differently in necrosis, inflammatory infiltration [16] and cancer stem cells [17]. As a result, intratumoral heterogeneity could be depicted in PET images through chaotic FDG uptake. As shown in our results, lower heterogeneity parameter correlated with larger MTV and advanced N stage. Similarly, one previous research of Kidd et al. also found that cervical pretreatment FDG-PET metabolic heterogeneity predict risk of lymph node involvement and pelvic recurrence [18]. Additionally, tumor recurrence observed in low AUC-CSH group was 3 times higher than high AUC-CSH group in our research.

The pretreatment larger MTV was associated with worse outcomes in certain cancers such as the lung [19], oral cavity carcinoma [20], and uterine cervical cancer [21] independent of disease stage. In this study, we also found smaller MTV $(\leq 13.6)$ was associated with improved RFS in univariate analysis. However, AUCCSH was the only independent predictor of RFS. Based on previous researches [7, 22], we know that tumor size was correlated with tracer uptake heterogeneity.

In another aspect, dynamic monitoring of MTV during treatment may be more valuable and practical in the clinic. Wei et al. found that a decrease of MTV during the early stage of CCRT was correlated with higher OS [23]. Tamandl et al. also concluded that volumetric changes induced by neoadjuvant chemotherapy are independent prognostic factors for survival in patients with radical esophageal cancer resection [24]. For R0 resection, MTV change can't be monitored in this research. However, we are conducting another prospective study to monitor more metabolic parameters' change for unresectable ESCC.

Unlike in previous reports [25-27] on the correlation of $\mathrm{SUV}_{\max }$ with treatment response and prognoses of various malignancies, we did not find any significance between $\mathrm{SUV}_{\text {max }}$ for the primary tumor and outcome in our cohort. Median value of $\mathrm{SUV}_{\max }$ was used as the optimal cut-off for analyses in this study. Nevertheless, the cut-off value of $\mathrm{SUV}_{\max }$ (14.9) was also estimated from ROC analysis in predicting tumor recurrence. Specificity (70\%, 95\% CI: $38.2 \%-89.6 \%)$ and sensitivity $(52 \%, 95 \%$ CI: $35.1 \%-70.2 \%)$ were derived from AUC-ROC (0.572, $P=0.073)$, it also failed to show statistical significance 
in analyses. Possible explanations for the discrepancy between the results of previous studies and the present study would be heterogeneity of tumor character and election of treatment. Therefore, the result of the present study on the predictive role of SUV must be interpreted with caution. Another explanation could be that because ESCC originate from the mucosa, the status of the mucosa (inflammation or postbiopsy) might increase maximal SUV. Interestingly, all these findings could make us speculate that AUC-CSH might be not a simple surrogate of SUV and could be an independent factor.

Although heterogeneity can be associated with malignant behavior of the tumor, there's no universal definition. Including visual evaluation [28], AUC-CSH [6], and texture analysis [4], a few main parameters have been proposed to quantify metabolic heterogeneity. Visual assessment may be considered as a simple way of scoring intratumoral tracer distribution. However, it can be difficult to implement in clinics because of higher interobserver variability. The accuracy and precision of texture analysis in clinical evaluation depends significantly on individual scanning protocols. Factors such as image acquisition, reconstruction and inherent image quality parameters may be important [29]. In comparison, the AUC-CSH index yields an intuitive and fairly robust tool for extracting information on the spatial gradient of the tumor heterogeneity as demonstrated by our results. Moreover, the sign of AUC-CSH index provides additional information on relative changes in SUV between the SUVbased tumor center and periphery.

The major disadvantages of current research are the retrospective design, the heterogeneity of the patients and treatments, and only primary tumor were analyzed. Besides, the process of ROI extraction was not full automation. Accurate segmentation technology could ensure the smooth implementation of multi-center studies. Ongoing studies will also explore the potential relationships between immunohistochemical staining patterns and intratumoural heterogeneity on functional imaging.

Despite the disadvantages, however, it was demonstrated that the preoperative intratumoral metabolic heterogeneity status characterized by AUC$\mathrm{CSH}$ was reliably to identify the high-risk population for postoperative recurrence and short survival in patients with radical ESCC resection. Large-scale investigations should be conducted to determine the bias and variance in multicenter. In conclusion, AUC-CSH could be an important factor to be considered in the treatment planning and follow-up of patients with resectable ESCC.

\section{MATERIALS AND METHODS}

\section{Patients}

This study was approved by the institutional review board at Shandong Cancer Hospital. Informed consent was waived due to the retrospective design of the study. From the cancer registry at Shandong Cancer Hospital, we retrospectively analyzed one hundred and sixteen consecutive patients with previously untreated, biopsyproven ESCC from March 2010 to March 2013. All the patients underwent esophagogastroduodenoscopy, endoscopic ultrasound, computed tomography scan of chest and upper abdomen, and 18F-FDG PET/CT scan before curative esophagectomy. For patients who have not received preoperative therapy, our institute has included postoperative fluoropyrimidine-based chemoradiation for patients with T3-T4 tumors, node-positive T1-T2 tumors, and selected patients with T2, N0 tumors with high-risk features. Locoregional recurrence was defined as occurring on an anastomosis site, the mediastinum, or the abdomen where lymph nodes were dissected. Distant recurrence was defined as those occurring outside the operative field, such as in the lung, brain, liver, adrenal glands, bone, or other location. Recurrence was diagnosed based on the $\mathrm{PET} / \mathrm{CT}$ and chest $\mathrm{CT}$ results, and tissue biopsies were taken of suspected recurrent lesions if possible. Adjuvant therapy including radiotherapy (RT) and chemotherapy (CT) after initial curative esophagectomy was determined after discussion among the surgical, medical, and radiation oncologists. Clinical follow-up was done every 2-4 months during the first year, every 4-6 months during the next 2 years, and every year thereafter.

\section{F-FDG PET/CT Scan}

All the 116 patients underwent the pre-treatment whole-body 18F-FDG PET/CT scan 1 week before the surgery. Patients were fasted for at least $8 \mathrm{~h}$ prior to 18F-FDG PET/CT scanning, and the blood glucose level was $<1.4 \mathrm{~g} / \mathrm{L}$ before scans for all patients. The FDG PET/CT images were obtained using a GE Discovery LS system 60 minutes (range 55-70 min) after injection of $18 \mathrm{~F}-\mathrm{FDG}(4.4 \mathrm{MBq} / \mathrm{kg})$ with a rigid protocol [30]. CT data were acquired first $(120 \mathrm{kV}$ and $90 \mathrm{~mA}$, no contrast enhancement). PET images were subsequently reconstructed with the built-in GE advance software, using the ordered subset expectation maximization (OSEM) algorithm with 2 iterations and 28 subsets, and a $5.0 \mathrm{~mm}$ full-width at half-maximum (FWHM) Gaussian postfiltering. The PET $(128 \times 128$, pixels of $3.91 \times 3.91 \mathrm{~mm}$, $4.25-\mathrm{mm}$ slice thickness $)$ and the CT $(512 \times 512$, pixels of $0.98 \times 0.98 \mathrm{~mm}, 5.0 \mathrm{~mm}$ slice thickness) images were systematically co-registered using the GE software.

\section{PET imaging analysis}

Our previous study demonstrated that the tumor length at FDG PET image with the cutoff value of 2.5 was closest to the gross tumor length [31]. Based on this result, the regions equal or greater than SUV 2.5 were selected to automatically delineate region of interest (ROI). Two 
clinical oncologists with the help of a specialist radiologist adjusted manually by visual inspection of the primary tumor borders to avoid overlapping on adjacent FDGavid structures or lesions. All metabolic parameters were subsequently extracted from this delineated volume. The SUVmax in each ROI was determined using the whole-body attenuation corrected image. The MTV was automatically generated from the ROI in cubic centimeters (cm3) using the Xeleris workstation.

Intratumoral metabolic heterogeneity was evaluated by the AUC-CSH, which was known to reflect the tumor heterogeneity [7, 13, 14]. These histograms are similar to dose-volume histograms frequently used in radiotherapy. $\mathrm{CSH}$ is normally obtained by plotting the percent volume of a tumor with an SUV above a certain threshold against that threshold, which is varied from 0 to $100 \%$ of SUVmax. The AUC of this plot (AUC-CSH) is a quantitative index of uptake heterogeneity, where lower values correspond with increased heterogeneity. Figure 2 shows two typical examples of FDG uptake heterogeneity, the metabolic tumor volume (black) was the ROI to be segmented and analyzed. All image processing process such as ROI segmentation, denoising and $\mathrm{CSH}$ extraction was performed using a code developed and implemented in-house at MATLAB (Mathworks Inc, Natick, USA).

\section{Study design and statistical analysis}

To evaluate the prognostic value of intratumoral metabolic heterogeneity feature, Relapse-free survival (RFS) and OS was chosen as endpoints. RFS was calculated from the date of the operation to the date of the first recurrence or the last follow-up. OS was measured from the date of the operation to the date of death or the last follow-up .The statistics analysis was performed using SPSS for Mac (version 22, IBM). According to clinical characteristics, the AUC-CSH of various groups was expressed as the mean $\pm \mathrm{SD}$. The differences of AUC-CSH among subgroup were tested using one-way analysis of variance (ANOVA) or Kruskal-Wallis test. The $\mathrm{SUV}_{\text {max }}$, MTV, AUC-CSH and ages were analyzed as binary variables using the median values as cutoff levels, which is more than median as the high group and less than or equal to median as the low group. Tumor (T) classification, lymph node $(\mathrm{N})$ classification, differentiated degree $(\mathrm{G})$ and AJCC stage were analyzed as categorical variables. Survival probabilities were estimated using the KaplanMeier method, and the difference between survival curves in relation to low and high levels of each prognostic factors were tested using the log-rank test in the univariate analysis. Multivariate analysis was performed to identify the prognostic factors influencing RFS and OS using Cox
A

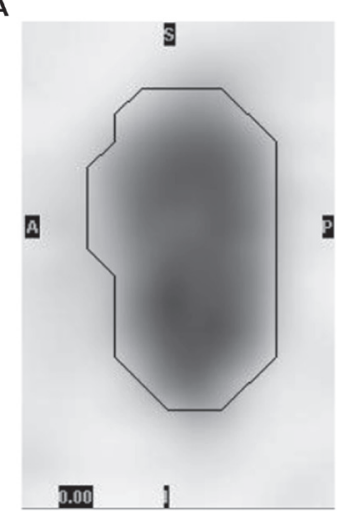

C

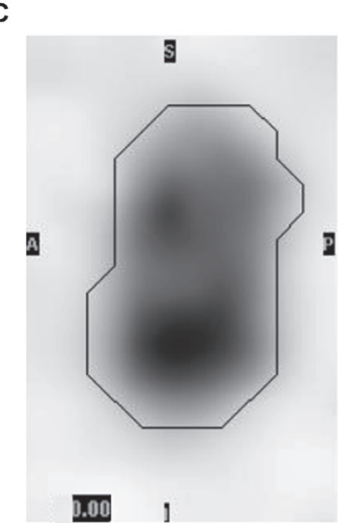

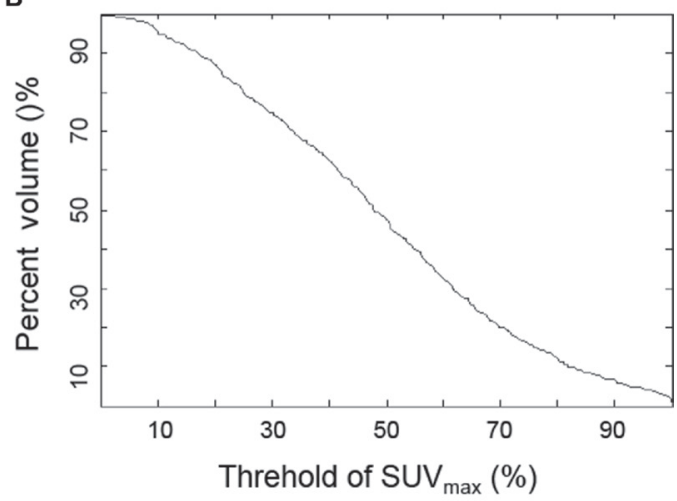

D

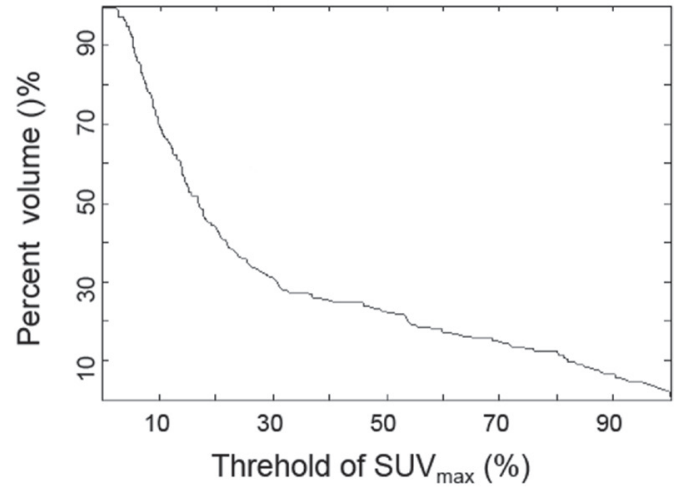

Figure 2: Typical examples of FDG uptake heterogeneity. Tumor with a lower degree of heterogeneity (A) showed higher AUC-CSH (B) and tumor with a higher degree of heterogeneity (C) showed lower AUC-CSH (D). 
proportional hazards regression model. Only significant variables after univariate survival analysis were included in multivariate survival analysis. All statistical tests were conducted at a two-sided level of significance of 0.05 .

\section{ACKNOWLEDGMENTS}

The authors thank Pro. Dengwang Li from College of Physics and Electronic Science, Shandong Normal University for the image analysis code development and implementation.

\section{CONFLICTS OF INTEREST}

The authors have declared that no conflicts of interests exist.

\section{GRANT SUPPORT}

This work was supported in part by the grant from the Health and Family Planning Commission of Shandong Province (2014WS0060), National Natural Science Foundation of China (81272502 and 81572970), Shandong Natural Science Foundation (ZR2014YL033) and the Educational fund from the China Scholarship Council (201406220074).

\section{REFERENCES}

1. Subramaniam D, Ponnurangam S, Ramamoorthy $P$, Standing D, Battafarano RJ, Anant S, Sharma P. Curcumin Induces Cell Death in Esophageal Cancer Cells through Modulating Notch Signaling. Plos One, 2012, 7:786-786. doi: 10.1371/journal.pone.0030590.

2. Pakzad R, Mohammadian-Hafshejani A, Khosravi B, Soltani S, Pakzad I, Mohammadian M, Salehiniya H, Momenimovahed Z. Incidence and mortality of oesophageal cancer and their relationship to development in Asia. Ann Transl Med. 2016; 4:29. doi: 10.3978/j.issn.23055839.2016.01.11.

3. Worni M, Martin J, Gloor B, Pietrobon R, D'Amico TA, Akushevich I, Berry MF. Does surgery improve outcomes for esophageal squamous cell carcinoma? an analysis using the surveillance epidemiology and end results registry from 1998 to 2008. Journal of the American College of Surgeons. 2012; 215:643-51. doi: 10.1016/j.jamcollsurg.2012.07.006.

4. Rahim MK, Kim SE, So H, Kim HJ, Cheon GJ, Lee ES, Kang KW, Lee DS. Recent Trends in PET Image Interpretations Using Volumetric and Texture-based Quantification Methods in Nuclear Oncology. Nuclear Medicine and Molecular Imaging. 2014; 48:1-15. doi: 10.1007/s13139-013-0260-2.

5. Chicklore S, Goh V, Siddique M, Roy A, Marsden PK, Cook GJR. Quantifying tumour heterogeneity in 18F-FDG $\mathrm{PET} / \mathrm{CT}$ imaging by texture analysis. European Journal of
Nuclear Medicine and Molecular Imaging. 2013; 40:133-40. doi: 10.1007/s00259-012-2247-0.

6. Van Velden FHP, Cheebsumon P, Yaqub M, Smit EF, Hoekstra OS, Lammertsma AA, Boellaard R. Evaluation of a cumulative SUV-volume histogram method for parameterizing heterogeneous intratumoural FDG uptake in non-small cell lung cancer PET studies. Eur J Nucl Med Mol Imaging. 2011; 38:1636-47. doi: 10.1007/s00259-011-1845-6.

7. Dong X, Wu P, Sun X, Li W, Wan H, Yu J, Xing L. Intratumour (18) F-FDG uptake heterogeneity decreases the reliability on target volume definition with positron emission tomography/computed tomography imaging. J Med Imaging Radiat Oncol. 2015; 59:338-45 . doi: 10.1111/1754-9485.12289.

8. Dong X, Xing L, Wu P, Fu Z, Wan H, Li D, Yin Y, Sun X, Yu J. Three-dimensional positron emission tomography image texture analysis of esophageal squamous cell carcinoma: relationship between tumor F-18-fluorodeoxyglucose uptake heterogeneity, maximum standardized uptake value, and tumor stage. Nucl Med Commun. 2013; 34:40-6. doi: 10.1097/MNM.0b013e32835ae50c.

9. Kang SR, Song HC, Byun BH, Oh JR, Kim HS, Hong SP, Kwon SY, Chong A, Kim J, Cho SG, Park HJ, Kim YC, Ahn SJ, et al. Intratumoral Metabolic Heterogeneity for Prediction of Disease Progression After Concurrent Chemoradiotherapy in Patients with Inoperable Stage III Non-Small-Cell Lung Cancer. Nucl Med Mol Imaging. 2014; 48:16-25. doi: 10.1007/s13139-013-0231-7.

10. Dong X, Sun X, Sun L, Maxim PG, Xing L, Huang Y, Li W, Wan H, Zhao X, Xing L, Yu J. Early Change in Metabolic Tumor Heterogeneity during Chemoradiotherapy and Its Prognostic Value for Patients with Locally Advanced NonSmall Cell Lung Cancer. PLoS One. 2016; 11:e0157836. doi: 10.1371/journal.pone.0157836.

11. Bedard PL, Hansen AR, Ratain MJ, Siu LL. Tumour heterogeneity in the clinic. Nature. 2013; 501:355-64. doi: 10.1038 /nature 12627 .

12. Ingelfinger JR, Rustgi AK, El-Serag HB. Esophageal Carcinoma. N Engl J Med. 2014; 371:2499-509. doi: 10.1056/NEJMra1314530.

13. van Baardwijk A, Bosmans G, van Suylen RJ, van Kroonenburgh M, Hochstenbag M, Geskes G, Lambin P, De Ruysscher D. Correlation of intra-tumour heterogeneity on 18 F-FDG PET with pathologic features in non-small cell lung cancer: a feasibility study. Radiother Oncol. 2008; 87: 55-8. doi: 10.1016/j.radonc.2008.02.002.

14. Gerlinger M, Rowan AJ, Horswell S, Larkin J, Endesfelder D, Gronroos E, Martinez P, Matthews N, Stewart A, Tarpey P, Varela I, Phillimore B, Begum S, et al. Intratumor Heterogeneity and Branched Evolution Revealed by Multiregion Sequencing. N Engl J Med. 2012; 366:883-92. doi: 10.1056/NEJMoa1113205.

15. Meacham CE, Morrison SJ. Tumour heterogeneity and cancer cell plasticity. Nature [Internet]. 2013; 501:328-37. doi: 10.1038/nature12624. 
16. Brown RS, Leung JY, Fisher SJ, Frey KA, Ethier SP, Wahl RL. Intratumoral distribution of Tritiated Fluorodeoxyglucose in Breast Carcinoma: Are Inflammatory Cells Important? J Nucl Med. 1995; 36:1854-61. Available from http://www.ncbi.nlm.nih.gov/ pubmed/7562055.

17. Vlashi E, Lagadec C, Vergnes L, Matsutani T, Masui K, Poulou M, Popescu R, Della Donna L, Evers P, Dekmezian C, Reue K, Christofk H, Mischel PS, et al. Metabolic state of glioma stem cells and nontumorigenic cells. Proc Natl Acad Sci USA. 2011; 108:16062-7. doi: 10.1073/pnas.1106704108.

18. Kidd EA, Grigsby PW. Intratumoral metabolic heterogeneity of cervical cancer. Clin Cancer Res. 2008; 14:5236-41. doi: 10.1158/1078-0432.CCR-07-5252.

19. Lee JY, Choi JY, Heo JH, Han J, Jang SJ, Kim K, Kim J, Shim YM, Kim B-T. Prognostic significance of volumebased 18F-FDG PET/CT parameter in patients with surgically resected non-small cell lung cancer. Comparison with immunohistochemical biomarkers. Nuklearmedizin. 2016; 55:7-14. doi: 10.3413/Nukmed-0754-15-07.

20. Zhang H. Abele, J. T. Jeffery, D. T. Harris, J. R. O'Connell, D. A. HS. Metabolic tumour volume as a prognostic factor for oral cavity squamous cell carcinoma treated with primary surgery. J Otolaryngol Head Neck Surg. 2016; 43:1-9. doi: 10.1016/j.oraloncology.2016.03.013.

21. Chung HH, Cheon GJ. Prognostic value of preoperative intratumoral FDG uptake heterogeneity in early stage uterine cervical cancer. J Nucl Med. 2015; 56:346. doi: 10.1016/j.ygyno.2015.01.174.

22. Hatt M, Cheze-le Rest C, van Baardwijk A, Lambin P, Pradier O, Visvikis D. Impact of tumor size and tracer uptake heterogeneity in (18)F-FDG PET and CT non-small cell lung cancer tumor delineation. J Nucl Med. 2011; 52:1690-7. doi: 10.2967/jnumed.111.092767.

23. Huang W, Fan M, Liu B, Fu Z, Zhou T, Zhang Z, Gong H, Li B. Value of metabolic tumor volume on repeated 18F-FDG PET/CT for early prediction of survival in locally advanced non-small cell lung cancer treated with concurrent chemoradiotherapy. J Nucl Med. 2014; 55:1584-90. doi: 10.2967/jnumed.114.143297\r10.2967/jnumed.114.142919.

24. Tamandl D, Gore RM, Fueger B, Kinsperger P, Hejna M, Paireder M, Haug A, Schoppmann SF, Ba-Ssalamah A. Change in volume parameters induced by neoadjuvant chemotherapy provide accurate prediction of overall survival after resection in patients with oesophageal cancer. Eur Radiol. 2016; 26:311-21. doi: 10.1007/s00330-015-3860-7.
25. Na F, Wang J, Li C, Deng L, Xue J, Lu Y. Primary tumor standardized uptake value measured on F18Fluorodeoxyglucose positron emission tomography is of prediction value for survival and local control in nonsmall-cell lung cancer receiving radiotherapy: metaanalysis. J Thorac Oncol. 2014; 9:834-42. doi: 10.1097/ JTO.0000000000000185.

26. Akkas BE, Vural GU. Standardized uptake value for $18 \mathrm{~F}$-fluorodeoxyglucose is correlated with a high International Prognostic Index and the presence of extranodal involvement in patients with Diffuse Large B-Cell Lymphoma. Rev Esp Med Nucl Imagen Mol. 2014; 33:148-52. doi: 10.1016/j.remn.2013.07.008.

27. Groheux D, Giacchetti S, Moretti JL, Porcher R, Espié M, Lehmann-Che J, De Roquancourt A, Hamy AS, Cuvier C, Vercellino L, Hindié E. Correlation of high 18F-FDG uptake to clinical, pathological and biological prognostic factors in breast cancer. Eur J Nucl Med Mol Imaging. 2011; 38:426-35. doi: 10.1007/s00259-010-1640-9.

28. Tixier F, Hatt M, Valla C, Fleury V, Lamour C, Ezzouhri S, Ingrand P, Perdrisot R, Visvikis D, Le Rest CC. Visual Versus Quantitative Assessment of Intratumor 18F-FDG PET Uptake Heterogeneity: Prognostic Value in Non-Small Cell Lung Cancer. J Nucl Med. 2014; 55:1235-41. doi: 10.2967/jnumed.113.133389.

29. Yan J, Chu-Shern JL, Loi HY, Khor LK, Sinha AK, Quek ST, Tham IWK, Townsend D. Impact of Image Reconstruction Settings on Texture Features in 18F-FDG PET. J Nucl Med. 2015; 56:1667-73. doi: 10.2967/ jnumed.115.156927.

30. Yu J, Li X, Xing L, Mu D, Fu Z, Sun X, Sun X, Yang G, Zhang B, Sun X, Ling CC. Comparison of Tumor Volumes as Determined by Pathologic Examination and FDG-PET/ CT Images of Non-Small-Cell Lung Cancer: A Pilot Study. Int J Radiat Oncol Biol Phys. 2009; 75:1468-74. doi: 10.1016/j.ijrobp.2009.01.019.

31. Zhong $\mathrm{X}, \mathrm{Yu} \mathrm{J}$, Zhang $\mathrm{B}, \mathrm{Mu} \mathrm{D}$, Zhang W, Li D, Han A, Song P, Li H, Yang G, Kong FM, Fu Z. Using 18F-Fluorodeoxyglucose Positron Emission Tomography to Estimate the Length of Gross Tumor in Patients With Squamous Cell Carcinoma of the Esophagus. Int J Radiat Oncol Biol Phys. 2009; 73:136-41. doi: 10.1016/j. ijrobp.2008.04.015. 